\title{
Injection and injection-compression moulding replication capability for the production of polymer lab-on-a-chip with nano structures
}

\author{
Calaon, M.; Tosello, G.; Garnaes, J.; Hansen, H. N.
}

Published in:

Journal of Micromechanics and Microengineering

Link to article, DOI:

10.1088/1361-6439/aa853f

Publication date:

2017

Document Version

Peer reviewed version

Link back to DTU Orbit

Citation (APA):

Calaon, M., Tosello, G., Garnaes, J., \& Hansen, H. N. (2017). Injection and injection-compression moulding replication capability for the production of polymer lab-on-a-chip with nano structures. Journal of Micromechanics and Microengineering, 27(10), [105001]. https://doi.org/10.1088/1361-6439/aa853f

\section{General rights}

Copyright and moral rights for the publications made accessible in the public portal are retained by the authors and/or other copyright owners and it is a condition of accessing publications that users recognise and abide by the legal requirements associated with these rights.

- Users may download and print one copy of any publication from the public portal for the purpose of private study or research.

- You may not further distribute the material or use it for any profit-making activity or commercial gain

- You may freely distribute the URL identifying the publication in the public portal 


\title{
Injection and injection-compression moulding replication capability for the production of polymer Lab-on-a-Chip with nano structures
}

\author{
M Calaon ${ }^{1}$, G Tosello ${ }^{1}$, J Garnaes² and H N Hansen ${ }^{1}$ \\ ${ }^{1}$ Department of Mechanical Engineering, Technical University of Denmark, Produktionstorvet, Building 427A, DK- \\ 2800, Kgs. Lyngby, Denmark \\ ${ }^{2}$ Danish Fundamental Metrology, Matematiktorvet, Building 307, DK-2800, Kgs. Lyngby, Denmark
}

E-mail: mcal@mek.dtu.dk

\begin{abstract}
The manufacturing precision and accuracy in the production of polymer Lab-on-a-Chip components with 100-130nm deep nanochannels are evaluated using a metrological approach. Replication fidelity on corresponding process fingerprint test nanostructures over different substrates (nickel tool and polymer part) is quantified through traceable atomic force microscope measurements. Dimensions of injection moulded (IM) and injection-compression moulded (ICM) thermoplastic cyclic olefin copolymer nanofeatures are characterized depending on process parameters and four different features positions on a 30x80 $\mathrm{mm}^{2}$ area. Replication capability of IM and ICM technologies are quantified and the products
\end{abstract} tolerance at the nanometre dimensional scale verified.

\section{Introduction}

The capability of fabricating at low cost and with high volume micro and nano structures into polymer substrates has experienced in the last decade a global increasing interest [1]. Hot embossing (HE), injection moulding (IM) and injection compression moulding (ICM) are recognized as among the most important methods to replicate micro/nano features on polymer [2][3][4].

Particularly the achievable accuracy to which the nano geometries are fabricated is key aspect. Replication of surface texture and topography at micro and nano scale are quantified and described using different rules and approaches [5]. Typically a ratio between tool master geometries and the produced part is expressed to quantify the replication quality of different technologies enabled by surface replication. The physical mirroring of the structures during replication introduces the need for comparable measurements (i.e. location of measuring points). At this dimensional scale the lower limit in terms of measurement precision (repeatability) and accuracy (calibration) are reached. As a consequence, the degree of replication dependencies from process condition, tool accuracy, material behaviour and features geometries should be directly correlated to the quantity intended to be measured. It is of central importance to establish clear boundary conditions on which production of nano technology-based product can be enabled by replication technologies. Measurement science and standards are key to provide regulation among international trade and to lower existing barriers in terms of technological challenges [1]. To find these boundaries, dimensional and geometrical metrology require establishing traceability of measuring results as an integrated part of product development, characterization and manufacture. A challenge is represented by the definition of product tolerances and its verification at nano scale [6][7][8][9]. The present work addresses these requirements. A metrological model that deals with the definition of replication fidelity based on dimensional and geometrical metrology is implemented. In an industrial context traceability of measuring results is key, therefore this particular study focus on calibration and establishment of traceability as an integrated part of the proposed measuring solutions.

In this paper, injection molding and compression molding processes capabilities are analyzed comparing experimental results of produced sub-micrometre structures, employed in Lab-on-a-chip (LoC) micronanofluidic devices. The introduction of characteristic geometries called process finger print, within the fabricated nickel shim, enabling product and process quality monitoring is presented. Nano channels gratings were defined as finger print test structures and molded on Cyclic Olefin Copolymer (COC) Topas 5013 L10. The injection molding process setting levels demonstrated in [10] to improve the replication quality of 
produced sub-micrometer geometries was used as starting point to define the new optimal process windows used during the molding experiments presented in this work. Replication fidelity between master and polymer geometries produced by conventional IM and ICM was quantified in terms of dimensional deviation from the corresponding nickel nano features. The proposed metrological approach allows for the possibility to quantify and compare, within an optimal process setup window, replication capability of the employed polymer processing technology, through calibrated atomic force microscope (AFM) measurements.

\section{Nano-structured tool manufacturing}

Based on developments obtained from silicon micro technology, lithography and etching were employed for the creation of different geometries. Electron beam lithography was used to define the nano structures whether photolithographic techniques, were employed to define micro structures using UV light source to activate photo sensible polymer resist covered/uncovered by a pre-patterned mask. Final etching defined depth of designed structures by removing material from silicon substrate. Mask material (silicon oxide for the proposed process chain) was used to selectively shadow the substrate material to be etched. Dry etching providing highly directional (anisotropic) etching of deep structures was employed in combination with reaction of plasma etching in the proposed dry etching electroplating and moulding (DEEMO) process as described in [11]. The presented process enables the production of a nickel shim by electrochemical deposition integrating different critical geometries of existing LoC designs for biomedical applications. Nano geometries produced over different positions of the nickel shim were subsequently revealed by etching the sacrificial silicon wafer (figure 1a). The central part of the shim is finally cut by wire electrical discharge machining to fit the $30 \mathrm{~mm} \times 80 \mathrm{~mm}$ active moulding area of the employed mould design. Nano structures produced on the nickel shim were transferred on the final disposable polymer devices (figure 1b) through large volume replication technologies, such as polymer moulding.

\subsection{Finger print nano features design}

In this study geometries designed for a nano fluidic system enabling single-molecule denaturation mapping of DNA [12] were manufactured and used to quantify replication capability of injection moulding and compression moulding processes.

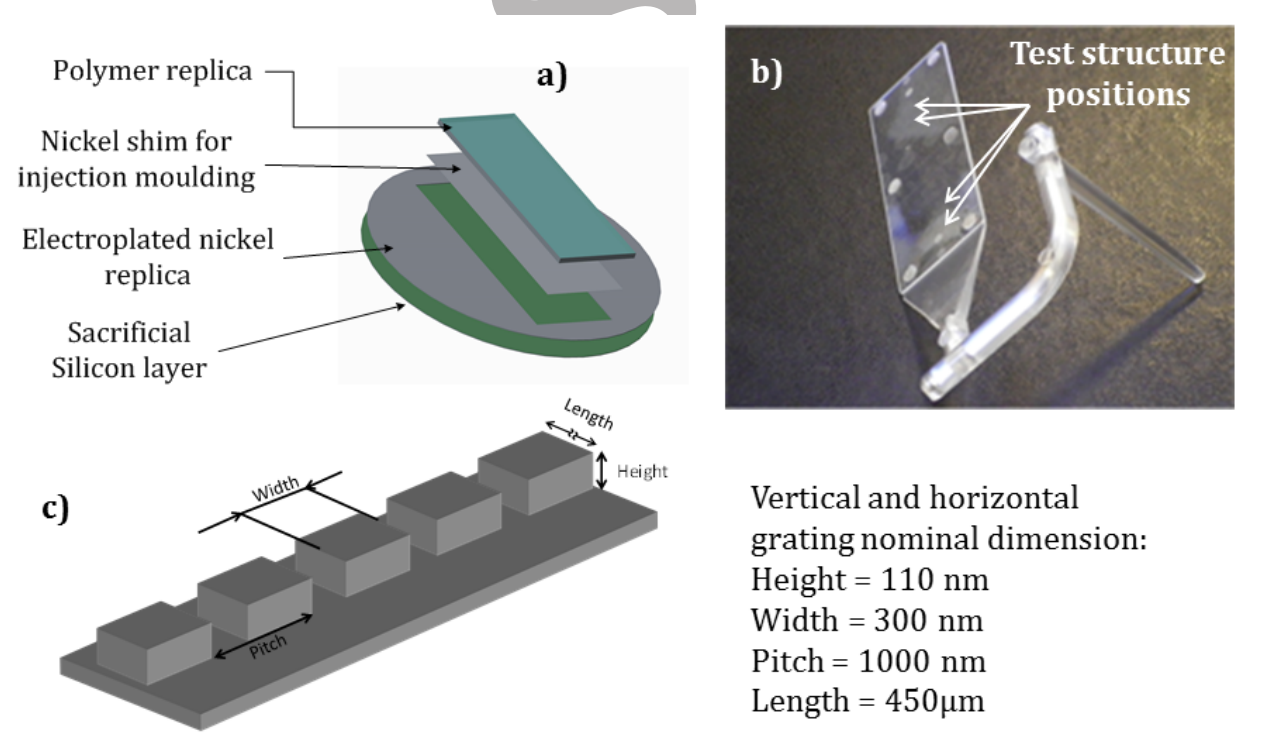

Figure 1: (a) Different process chain replication substrates for surface nano manufacturing; (b) actual injection moulded part integrating finger print test structures; (c) nano channels gratings nominal dimensions.

The nano channel grating on which DNA molecules are gathered for final optical analysis were identified as the critical features giving product functionalities. Therefore to obtain an effective product and process 
monitoring process finger print geometries (see figures 2a-d) were manufactured reproducing the same critical features of the functional LoC device bio-sensing application. The identified critical geometries and dimensions, figure 1c, of the LoC's nano channels were manufactured at different distances from the injection gate, figure 1b. With the introduction of these process-sensitive and representative finger print features, the inspection effort can be more efficient and effective by establishing the relation between nano moulded product quality parameters and the finger print quality response. In addition, product quality is increased by ensuring measurement traceability and measurement relocation.

\section{Measurement uncertainty}

Quality control of nano-objects is a key issue for product validation. Typically, in precision engineering oriented to large volume production, the measuring time and generally the inspection effort required to assess critical structures quality heavily influences the final product costs. These requirements are not trivial in nanomanufacturing especially if measurement time has to be comparable to part production time. Considering current state of the art of commercially available optical instruments lateral resolution is the main limiting factor for effective in-line quality control of nanostructured polymer surfaces. Current studies [13], [14] focus on new approaches to enable fast and robust characterization of textured surfaces in the nano meter range. AFM works in this scenarios as key reference techniques towards measurements traceability and optical system measurements capabilities verification. Uncertainties involved in the measuring procedure and instrument bias errors must be smaller than the product dimensional and topographical variations to perform robust and reliable analysis. For these reasons a calibrated AFM (NX20, manufactured by Park Systems) was used to assess and quantify the replication quality of produced functional and test structure geometries over the different substrates (i.e. from the nickel shim to the polymer part). In the AFM used the scanning tip is mounted on a separate z-flexure stage equipped with strain gauge distance sensors for accurate surface height measurement. The sensitivity of the strain gauge distance sensors is calibrated traceable to a step height standard which was calibrated by a metrological large range scanning probe microscope equipped with three stabilized laser interferometers ultimately traceable to the SI-unit metre [15] and [16].

The evaluation of the height deviation between polymer channels depth and the respective nickel trenches height is defined as $h_{\mathrm{dev}}$, see equation (1). This measured quantity ( $h_{\mathrm{dev}}$ ) gives the replication fidelity as the deviation of the polymer feature depth $h_{\text {pol }}$ from the corresponding height of the nickel master $h_{\text {nickel }}$.

Accuracy, precision of measuring results and measuring time were considered in order to find an optimal setup for the measurement campaign. Scanning areas of $10 \mu \mathrm{m} \times 10 \mu \mathrm{m}$ were performed over nickel and polymer substrates on the same characteristic features in order to calculate $h_{\mathrm{dev}}$ while minimizing the influence of relocation on different substrates.

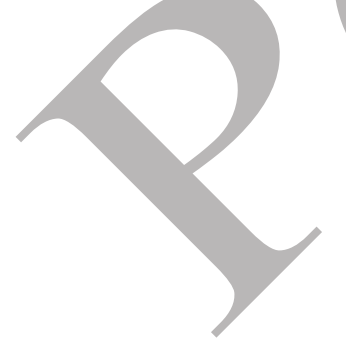


(a)

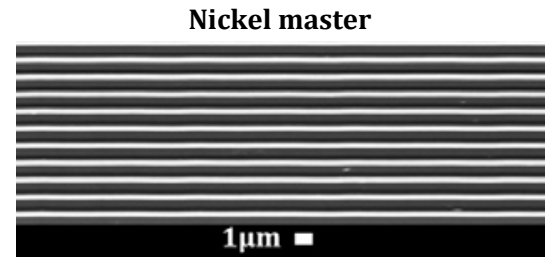

(c)

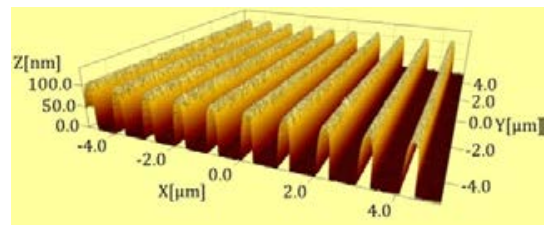

(e)

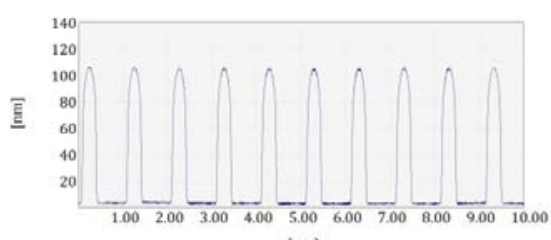

(b)

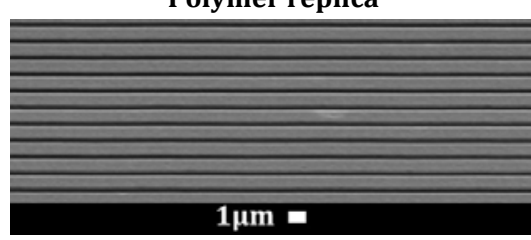

(d)
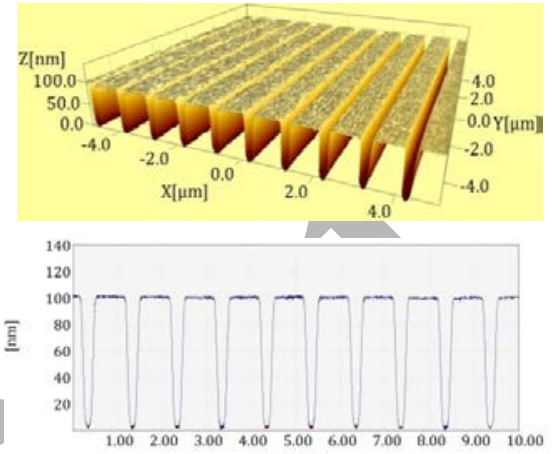

[um]

Figure 2: Finger print nano channels grating manufacture and dimensional characterization: (a), (b): SEM images of nickel and polymer produced test structures; (c), (d): 3D view of AFM scanned area on the two different substrates (evaluation area $10 \mu \mathrm{m} \times 10 \mu \mathrm{m})$; (e), (f): corresponding 2D step height profile analysis following calculation described in the ISO 5436.

Channels and trenches heights were analysed using a commercially available scanning probe processing software [17] applying for each scanned structure the ISO 5436 profile analysis procedure [18] (figures 2e and 2f). Errors contributors composing the combined uncertainty were quantified following the guide to the expression of uncertainty in measurements (GUM) [19]. In particular, type B uncertainty evaluation was calculated for uncorrelated (independent) input quantities identified. The model function for the measured quantity is

$$
h_{\mathrm{dev}}=\left(h_{\mathrm{pol}}-h_{\text {nickel }}\right)
$$

where the two terms $h_{\text {pol }}$ and $h_{\text {nickel }}$ are the observed height or depth of the polymer and nickel features calculated as:

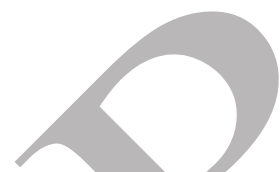

$$
\begin{gathered}
h_{\text {pol }}=\left(C_{z} \times h_{\text {obs, pol }}\right) \\
h_{\text {nickel }}=\left(C_{z} \times h_{\text {obs, nickel }}\right)
\end{gathered}
$$

where $C_{z}$ is the linear correction factor for the sensitivity of the distance sensors in the vertical z-direction. The linear correction factor $C_{z}$ is measured as the ratio of:

$$
C_{z}=\left(\frac{\Delta h_{\text {step, obs }}}{\Delta h_{\text {step,cert }}}\right)
$$

where $\Delta h_{\text {step,obs }}$ is the observed step height of the step height standard and $\Delta h_{\text {step,cert }}$ is the "true” step height of the step height standard measured by laser interferometers as described above. The combined standard uncertainty evaluation based on (1) is calculated as: 


$$
u_{c}^{2}\left(h_{\mathrm{dev}}\right)=\left(\frac{\partial h_{\mathrm{dev}}}{\partial C_{z}}\right)^{2} \times u^{2}\left(C_{z}\right)+\left(\frac{\partial h_{\mathrm{dev}}}{\partial h_{\mathrm{obs}, \mathrm{pol}}}\right)^{2} \times u^{2}\left(h_{\mathrm{obs}, \text { pol }}\right)^{2}+\left(\frac{\partial h_{\mathrm{dev}}}{\partial h_{\text {obs, nickel }}}\right)^{2} \times u^{2}\left(h_{\text {obse, nickel }}\right)^{2}
$$

The most influence parameters and their size are briefly discussed below:

- $u\left(C_{z}\right)$ is the uncertainty of to the linear correlation factor. This is influenced by $u_{\text {res }}=$ vertical instrument resolution; $u_{c, z}=$ variability of the calibration factor calculated as standard deviation of repeated independent measurements on calibrated artefact; $u_{\text {cal }}=$ calibration uncertainty of reference artefact, a calibrated step height standard reference [20]; $u_{\text {noise }}=$ instrument background noise. The uncertainty for $u\left(C_{z}\right)$ is estimated to be 0.02 .

- $u_{\mathrm{AFM}, \mathrm{z}}=$ is estimated as experimental standard deviation of 5 repeated measurements on the same manufacture nano feature (whether nickel or polymer).

- $u_{\text {features }, z}=$ is estimated as experimental standard deviation of repeated measurements on 5 different features on the same scanning area to estimate feature reproducibility in terms of master making and polymer replication.

- $u_{\text {sample, },}=$ is estimated as experimental standard deviation of repeated measurements on 5 different features on 5 different scanning areas.

Since repeated measurements on the same scanning area as well as on different areas of the components are also affected by the instrument repeatability itself, the largest contributor related to instrument repeatability $\left(\mathrm{u}_{\mathrm{AFM}, \mathrm{z}}, \mathrm{u}_{\text {features }, \mathrm{z}}, \mathrm{u}_{\mathrm{sample}, \mathrm{z}}\right)$ was selected to not overestimate the effect of instrument repeatability and re-location [21]. Based on this and considering the uncertainty contribution given by the first term of (5) the following is obtained:

$$
\left(\frac{\partial h_{d e v}}{\partial C_{z}}\right)^{2} \times u^{2}\left(h_{o b s, d e v}\right)^{2}=\left(u_{o b s, p o l}^{2}-u_{o b s, n i c}^{2}\right)^{2} \times u_{C_{z}}^{2} \approx 0
$$

resulting in the final $\mathrm{U}_{\text {hdev }}$ expression:

$$
U_{\text {hdev }}=k \times C_{z} \times \sqrt{u_{o b s, p o l}^{2}+u_{o b s, \text { nic }}^{2}}
$$

where coverage factor $\mathrm{k}=2$, for a confidence level of $95 \%$.

\begin{tabular}{|c|c|c|c|c|}
\hline \multicolumn{2}{|r|}{$u_{j}$ contributors } & Nickel shim & \multirow{2}{*}{$\begin{array}{c}\begin{array}{c}\text { Polymer sample } \\
\text { IM }\end{array} \\
0.28\end{array}$} & \multirow{2}{*}{$\begin{array}{c}\begin{array}{c}\text { Polymer sample } \\
\text { ICM }\end{array} \\
\\
0.34\end{array}$} \\
\hline $\bar{\Xi}$ & $\mathrm{u}_{\mathrm{afm}, \mathrm{z}}$ (instr. repeatability) & 0.14 & & \\
\hline 善 & $\mathrm{u}_{\text {features,z }}$ (feat. repeatability) & 0.60 & 0.43 & 0.46 \\
\hline$=$ & $\mathrm{u}_{\text {sample,z }}$ (samp. repeatability) & 0.64 & 0.57 & 0.54 \\
\hline & $\mathrm{U}_{\text {hdev }}$ & 1.3 & 1.7 & 1.7 \\
\hline
\end{tabular}

Table 1: Uncertainty of vertical (i.e. height and depth) measurements of nano features on nickel shim and COC polymer replica produced by IM and ICM. Uncertainty contributors and expanded combined uncertainties are expressed in [nm]. 


\section{Moulding processes}

The nickel shim with active moulding area of $30 \mathrm{~mm} \times 80 \mathrm{~mm}$ was mounted on the movable half of the mould and assembled with an adaptable window containing runner and gate, see figure 3 . In the current study, a mould equipped with an oil heating system was employed. The moulding equipment adopted for the study is a full electric injection machine with screw diameter of $18 \mathrm{~mm}$ and maximum clamping force of $600 \mathrm{kN}$. On the same machine both IM and ICM were run. Both processes replicate positive or negative geometries manufactured directly in one or both half of the mould or else in interchangeable shims of different materials (nickel in the present case) fixed in one half of the mould. The material melts under the effect of the created friction of the screw and heater bands all along the injection cylinder. During the metering phase the screw with the rotary movement push the polymer in front of the screw tip, subsequently the melt polymer is injected by the screw pushed forward pressurizing the melt in front of the screw into the mould hydraulically closed at the beginning of the cycle. The ICM process is characterized by the same process cycle operations as in IM with the difference that a compression phase is added at the end of the injection phase. In ICM the two mould halves are kept at a certain distance during the injection phase allowing the polymer melt to flow inside the mould cavity. At the switch-over point the machine applies the clamping force and closes the two halves of the mould sealing the cavity. By establishing the compression phase a more uniform distribution of the cavity pressure is obtained while the polymer completes the filing phase.

Machine repeatability tests for both the configurations used during the experimental phase were carried out to verify process variation in terms of injection pressure. In particular the process parameter set for the data acquisition were:

- Injection moulding (IM): mould temperature $\left(125^{\circ} \mathrm{C}\right)$; melt temperature $\left(265^{\circ} \mathrm{C}\right)$; injection speed $(150$ $\mathrm{mm} / \mathrm{s}$ ); packing pressure (500 bar).

- Compression moulding (ICM): differently from the injection moulding the only parameter that had to be decreased to $115^{\circ} \mathrm{C}$ was the mould temperature not significantly influencing the maximum value of the injection pressure. Compression gap $(0.5 \mathrm{~mm})$ and switch over point $(13 \mathrm{~mm})$ were kept constant during the acquisition.

Acquisitions started after the machine was stabilized over the set process parameters values (approximately 25 moulded parts). Average maximum injection pressures (i.e. of switch over point from filling to packing phase) were $633 \pm 3$ bar and $581 \pm 2$ bar for injection moulding and compression moulding respectively.

Dimensional variation of 10 parts produced during machine repeatability tests for IM and 10 parts produced by ICM were assessed measuring test structures in position one (TS1) on both vertical and horizontal gratings. Each TS1 (vertical and horizontal gratings) were measured in 3 different location of $5 \mu \mathrm{m} \times 5 \mu \mathrm{m}$. The variability of TS1 dimensional variation calculated as standard deviation of repeated independent measurements was equal to $1.8 \mathrm{~nm}$ and $2.6 \mathrm{~nm}$ for IM and ICM respectively.

\subsection{Injection moulding}

The experimental IM phase was designed following results obtained in [10] that identified optimal process settings to replicate deterministic geometries in the sub-micrometre meter range. High mould temperature $\left(130^{\circ} \mathrm{C}\right)$, melt temperature $250^{\circ} \mathrm{C}$, high injection speed $(150 \mathrm{~mm} / \mathrm{s})$ and high level of packing pressure (600 bar) were demonstrated to decrease product dimensional variation and improve replication quality. The listed settings were used as initial reference to identify the new optimal process window for current study. The process levels were finally set according to the following: 
- Mould temperature levels were set at $115^{\circ} \mathrm{C}, 120^{\circ} \mathrm{C}, 125^{\circ} \mathrm{C}$, following material supplier recommendation and to allow micro and nano feature replication, complete part filling of the cavity at the selected high injection speed and to allow demoulding of the part from the cavity within acceptable cycle time.

- Melt temperature $255^{\circ} \mathrm{C}$ and $265^{\circ} \mathrm{C}$ was chosen as balanced value to avoid material degradation, and premature solidification during the filling of the cavity.

- Packing pressure levels were set at 500 bar and 600 bar allowing acceptable shrinkage compensation, dimensional accuracy, automatic demoulding without distortion, ensuring highest packing of the part and avoiding any over packing effect.

- Injection speed $150 \mathrm{~mm} / \mathrm{s}$ and $200 \mathrm{~mm} / \mathrm{s}$ considering the polymer capability to fill the cavities and replicate nano features before the melt flow solidifies, induced shear stress on polymer physical and optical properties; poor venting effectiveness resulting in diesel effect.

During the IM design of experiment (DOE) phase, 4 process parameters have been controlled in order to determine their influence on the surface replication. A general factorial design has been carried out performing a total of $3 \times 2^{3}=24$ moulding experiment combinations.

\begin{tabular}{cccc} 
Table 2: General factorial design with $3 \times 2^{3}=24$ experiments. \\
\hline $\begin{array}{l}\text { Process } \\
\text { Parameters }\end{array}$ & Levels \\
\hline & & & 125 \\
\cline { 3 - 4 } & 115 & 120 & \\
Mould temperature $\left[{ }^{\circ} \mathrm{C}\right]$ & 500 & 600 & \\
Packing pressure $[\mathrm{bar}]$ & 150 & 200 & \\
Injection speed $[\mathrm{mm} / \mathrm{s}]$ & 255 & 265 & \\
Melt temperature $\left[{ }^{\circ} \mathrm{C}\right]$ & &
\end{tabular}

Final measuring set up considered possible implementation in a production environment where trade-off between accuracy, precision of measuring results and especially scanning time are very relevant. Based on the preliminary machine tests and high dimensional accuracy measured over different samples sampling procedure was introduced to assess dimensional quality of produced samples. For each setting of the DOE 20 mouldings were run to obtain a stable and continuous process. After, 10 parts for each DOE combination were moulded and one sample for each DOE setting was randomly selected and measured. The replication quality of the produced features quantified as described in (1) was evaluated for the two different grating directions (i.e. parallel to flow and perpendicular to flow) at different distances from the gate position (TS1, TS2, TS3, TS4).

\subsection{Compression moulding}

The same insert with fabricated sub- $\mu \mathrm{m}$ structures was employed to produce the polymer parts. The optimization study performed using the same polymer COC 5013 for the IM process was used to establish the reference setting for the ICM experiments. The IM process setup that ensured features replication, acceptable cycle time and effective demoulding was selected (mould temperature $=115^{\circ} \mathrm{C}$, melt temperature $=265^{\circ} \mathrm{C}$, injection speed $=150 \mathrm{~mm} / \mathrm{s}$, packing pressure $=500 \mathrm{bar}$ ).

After partial filling of the cavity during the injection phase, the compression phase starts by applying the machine clamping force. The clamping force application was dependent and controlled by the screw position. During the experiments two main process parameters of injection compression were selected (based on [22], [23], [24]) and investigated: switch-over point (change point from velocity-controlled phase to pressurecontrolled phase, that here represents the starting of the compression phase) and mould opening distance.

Effects of moulding conditions on the replication fidelity of the test structures at different distances from the gate were quantified. A $3^{2}$ (3-levels, 2-factors) full factorial design has been carried out performing 9 moulding experiment combinations.

As in the case of the injection moulding experiments, for each setting of the ICM DOE 20 mouldings were run to obtain a stable and continuous process. After that, 10 parts for each DOE combination were produced and 
one sample for each setting was randomly selected and measured. The different process settings were established considering process feasibility and final part quality. In particular the process levels were set according to the following:

- Compression gap levels were set at $0.5 \mathrm{~mm}, 0.7 \mathrm{~mm}$, and $1 \mathrm{~mm}$ to ensure complete part filling and absence of part defect (e.g. diesel effect due to possible air entrapment, at the selected injection speed of $150 \mathrm{~mm} / \mathrm{s}$, presence of flash in the part).

- Switch over point levels were set at $12 \mathrm{~mm}, 16 \mathrm{~mm}, 20 \mathrm{~mm}$, to ensure acceptable shrinkage compensation, dimensional accuracy, automatic demoulding without distortion and to avoid any flash formation during the compression phase when clamping force was applied.

\section{Discussion and results}

The results from the injection moulding experiments are shown in the form of main effect plots (figure 3). Differences between outputs for different level settings of the considered factors are compared. Hence, the effects of different responses affecting the replication quality (height deviation) of produced structures are represented. Results from the injection moulding experiments, figure 3, show that higher mould temperature $\left(125^{\circ} \mathrm{C}\right)$ enhances replication fidelity between the nickel insert geometries and the transferred test structures geometries.

Close to the gate position, the effect of different mould temperatures on final replication quality are less significant due to the fact that this is the zone where the polymer solidifies last, i.e. where even a lower mould temperature is effective in promoting high replication fidelity. On the other hand, mould temperatures become a critical parameter for longer flow length. High mould temperature is required to improve replication for test structure positions far from the gate. Packing pressure presents similar trends for the measured test structures at different distances from the gate. More significant effect of the packing pressure is observed on test structures at increasing distance from the gate. Lower packing pressure resulted in being more effective in ensuring higher replication of the structures dimension. The higher level of packing pressure results in a decrease of features replication. This phenomenon suggests that a limit exists to packing pressure level (even before flash on the part occurs) due to increasing mould deflection occurring at high packing pressure settings [25].

Further, considerations can be made observing the effect of injection speed on the part dimensional deviation from the original master geometries. Injecting at higher speed would allow the polymer to flow for longer distances inside the mould cavity at higher temperatures giving a more effective filling even at a nanometre level where polymer viscosity play an important role [26]. However, a lower replication can be obtained at high injection speed settings due to limited air evacuation from the cavity [27]. Measurement results show that feature replication, for the employed polymer, gate dimensions and part geometry, degrades at high injection speed $(200 \mathrm{~mm} / \mathrm{s})$, limiting its potential advantage to improve the replication fidelity.

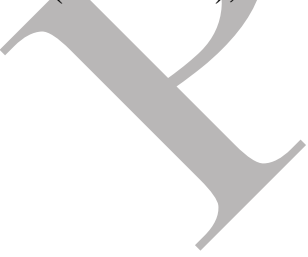




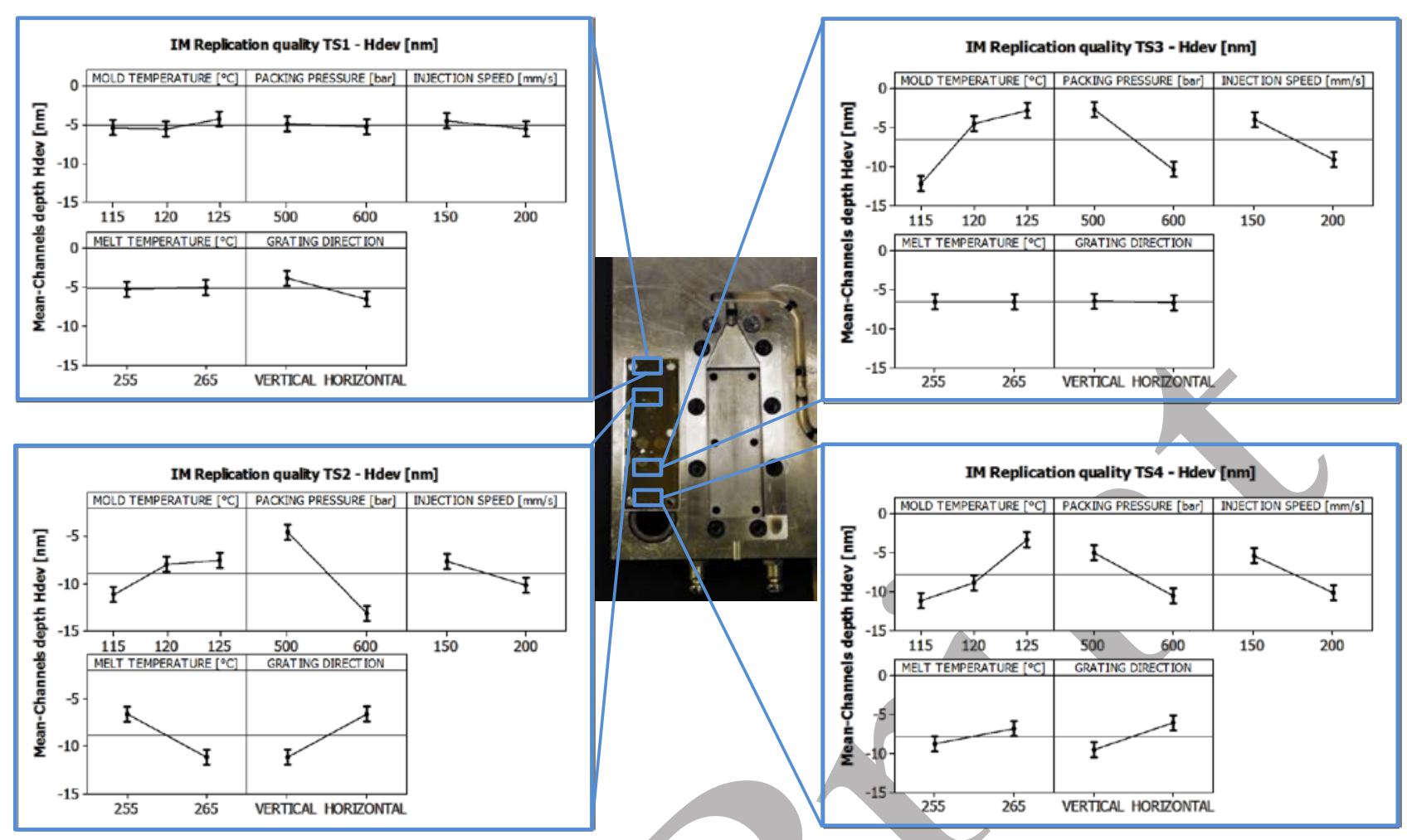

Figure 3: Main effect plot for polymer geometries produced by injection moulding depending on process parameters (mould temperature, packing pressure, injection speed, melt temperature) and part parameters (grating features direction, distance from the gate). Plots refer to effect of process parameters on the replication quality of the different test structures for different positions. Bars indicate combined standard uncertainty U. Average height of nickel test structures (TS) for the different positions: 135.1 $\pm 1.3 \mathrm{~nm}$ (TS1);

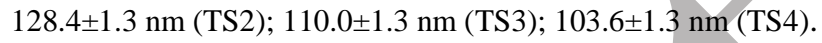

Compression moulding experiments are presented in figure 4. The compression gap has a significant consequence on the polymer geometries final quality on different test structure positions.

Generally in the two test structure positions closer to the gate (i.e. positions 1 and 2) feature dimensional variation is larger (max hdev $=16 \mathrm{~nm}$ ) than the one measured on positions far from the gate (i.e. position 3 and 4, max hdev $=8 \mathrm{~nm}$ ). Moreover, important indications from the measurement results are given by the comparison between the different compression gap effects on test structures position 2 and 4 . By increasing the compression gap in position 2 the feature replication increases, differently from what happens in position 4, where for a compression gap of $1 \mathrm{~mm}$ the product dimensional fidelity from the master geometries dimensions decreases. The lower replication degree at position 2 when applying a $0.5 \mathrm{~mm}$ gap before compression, is due to reduced air evacuation from in the mould which limits the feature replication. Further, the decreased feature replication measured in position 4 in correspondence of $1 \mathrm{~mm}$ gap is due to polymer premature cooling, i.e. to its higher viscosity. In fact, a larger compression gap causes a higher heat dissipation which promotes a decrease of melt temperature, hence increasing the melt viscosity.

Additionally, results show that the switch-over point (regulating the amount of material entering the cavity prior compression as well as the moment at which the compression phase starts) has a significant effect on the replication quality of investigated geometries. Especially for positions 1 and 4 an earlier switch-over point is effective in promoting feature replication in the compression phase. The gratings orientation with respect to the flow direction has shown smaller deviation for the horizontal features than for the vertical features in both IM and ICM. Features variation up to $5 \mathrm{~nm}$ and $10 \mathrm{~nm}$ were measured for IM and ICM respectively. 


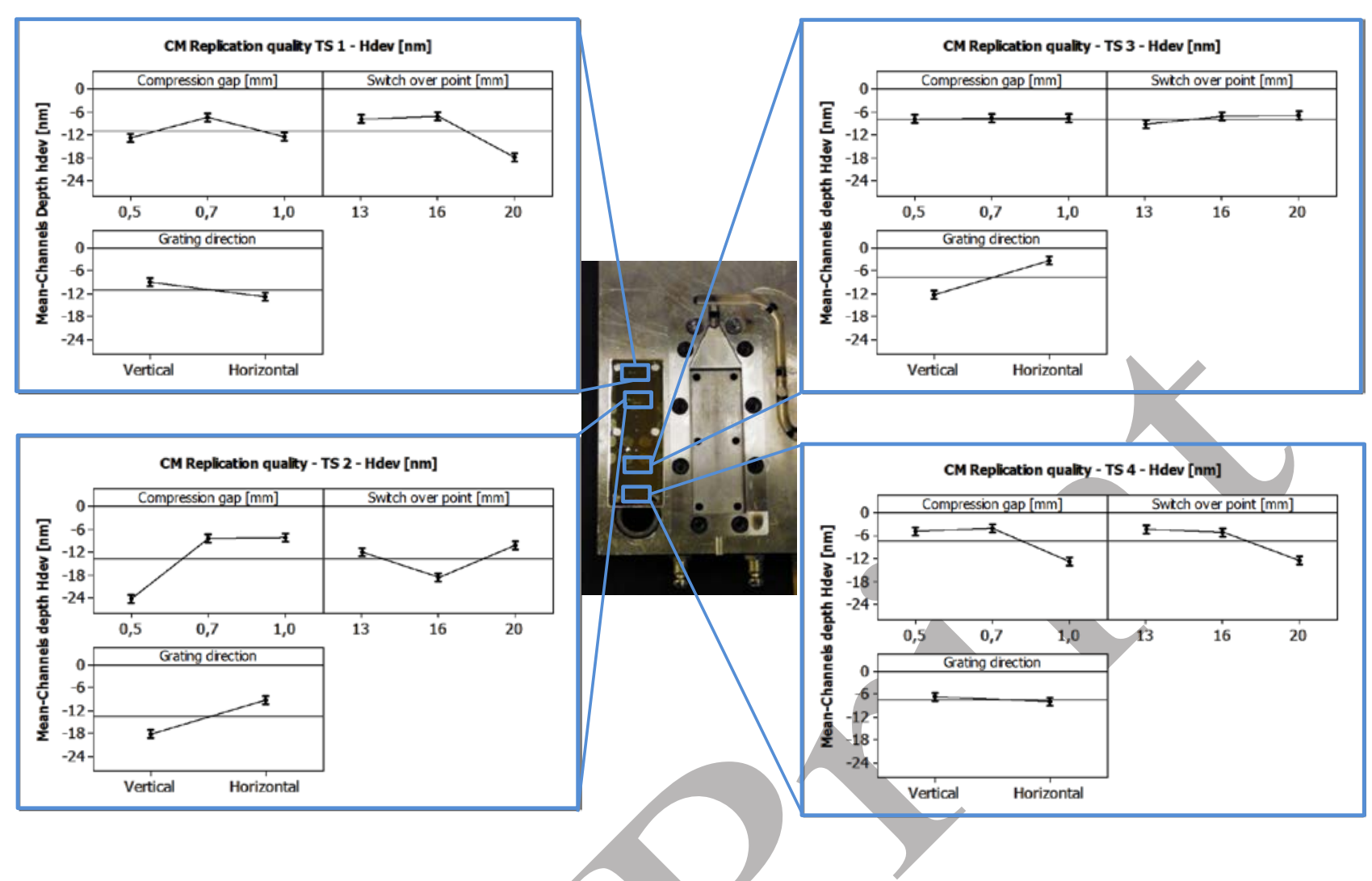

Figure 4: Main effect plot for polymer geometries produced by injection compression moulding depending on compression parameters (compression gap, switch over point) end part parameters (grating features direction, distance from the gate). Plots refer to effect of different process parameters on the replication quality of the different test structures for different positions. Bars indicate combined standard uncertainty U. Average height of nickel test structures (TS) for the different positions: $135.1 \pm 1.3 \mathrm{~nm}$ (TS1); $128.4 \pm 1.3 \mathrm{~nm}$ (TS2); $110.0 \pm 1.3 \mathrm{~nm}$ (TS3); $103.6 \pm 1.3 \mathrm{~nm}$ (TS4).

\section{Conclusion}

The accuracy and precision of injection and injection compression moulding processes were established to validate the process chain for the fabrication of nano structured polymer surfaces. Test structures designed in the form of nano channels gratings parallel and perpendicular to the flow direction, were introduced with the purpose of monitoring the production process.

The formulation of the uncertainty values for uncorrelated input quantities has been presented. Traceability of measurements results was ensured by calibrated AFM measurements. DOE optimization procedures have been carried out for both moulding processes measuring the height deviations of replicated test structures compared with nickel master geometries dimensions.

As far as the investigated injection moulding process windows is concerned, results of the analysis showed that high mould temperature $\left(125^{\circ} \mathrm{C}\right)$, high melt temperature $\left(265^{\circ} \mathrm{C}\right)$, low level of the injection speed $(150$ $\mathrm{mm} / \mathrm{s}$ ) and low level of packing pressure (500 bar) decreased deviations of polymer features from their corresponding features in the nickel mould i.e. improved the replication quality.

A further process characterization was performed for the compression moulding process. Replication quality assessment of the produced polymer features indicates that different compression gaps caused larger (16 nm) structures dimensional variation for feature positioned close to gate than structures positioned far from the gate $(8 \mathrm{~nm})$. Different compression gaps have significant effect on the replication of the produced nano features. In particular, with $0.5 \mathrm{~mm}$ compression gap, limited air evacuation from the mould during the compression phase could be achieved, negatively affecting the test structure replication in position near to the gate. Also, polymer higher viscosity due to cooling caused by larger $(1 \mathrm{~mm})$ compression gap, lower replication fidelity for test structures position at the end of the cavity. 
The finger print test structure concept has been validated to achieve process calibration through traceable measurements for the verification of surface sub- $\mu$ m structures replication fidelity. On this respect, the capability of quantifying process-and product-induced deviations in the single digit nanometre dimensional range of the moulded nano features was demonstrated.

\section{Acknowledgements}

The present research was carried out within the National Danish Strategic Research Centre PolyNano (http://www.polynano.org/) supported by the Danish Council for Strategic Research (DSF grant agreement no. 10-092322) and the European Innovative Training Network MICROMAN (http://www.microman.mek.dtu.dk/) (Process Fingerprint for Zero-defect Net-shape MICROMANufacturing) funded by the Marie Sklodowska-Curie Actions (MSCA) under the European Union's Horizon 2020 Research and Innovation Programme (grant agreement $n^{\circ}$ 674801). The collaboration of the Danish Metrology Institute (DFM) and the Scanning Probe Microscopy Group is greatly acknowledged.

\section{References}

[1] American National Science, Technology Council Committee on Technology Subcommittee on Nanoscale Science Engineering, and Technology 2014 National Nanotechnology Initiative Strategic Plan, , Tech.report, US Government, pp. 88.

[2] Heckele M and Schomburg W K 2003 Review on micro moulding of thermoplastic polymers, Journal of Micromech and Microeng, 14: R1 - R14.

[3] Peng L, Deng Y, Yi P and Lai X 2013 Micro hot embossing of thermoplastic polymers: a review Journal of Micromech and Microeng, 24:23 pp.

[4] Tosello G, Hansen N H, Calaon M and Gasparin S 2014 Challenges in high accuracy surface replication for micro optics and micro fluidics manufacture Precision Technology 4:122-144.

[5] Hansen H N, Hocken R J and Tosello G 2011 Replication ofmicro and nano surfaces Annals of the CIRP 60/2:695-714.

[6] Knapp W 2011 Tolerance and uncertainty Laser metrology and machine performance, pages 357-366.

[7] Kunzmann H, Pfeifer T, Schmitt R, Schwenke H and Weckenmann A 2006, Productive metrology - adding value to manufacture Annals of the CIRP 55/2:643-666.

[8] Weckenman A and Ernst R 2000 Studies on new tolerancing rules for micro and nanotechnology 1st euspen conference, 1:214-221.

[9] Hansen H N, Carneiro K, Haitjema H and De Chiffre L 2006, Emerging trends in surface metrology, Annals of the CIRP 55/2:721-743.

[10] Calaon M, Hansen H N, Tosello G, Garnæs J, Nørregaard J and Li W 2013 Microfluidic Chip Designs Process Optimization And Dimensional Quality Control, Journal of Microsystem Technologies, (DOI) 10.1007/s00542-013-2025-3., pp. 10

[11] Tanzi S, Ostergaard P F, Matteucci M, Christensen T L, Chech J, Marie R. and Taboryski R 2012 Fabrication of combined - scale nano- and microfluidic polymer systems using a multilevel dry etching, electroplating and molding process. Journal of Micromech and Microeng 22: pp. 11.

[12] Mikkelsen M B, Reisner W, Flyvbjerg H and Kristensen A 2011 Pressure-Driven DNA in Nanogroove Arrays: Complex Dynamics Leads to Length- and Topology-Dependent Separation Nano Letters 11:15981602.

[13] Calaon M, Madsen M H, Weirich J , Hansen H N, Tosello G, Hansen P E, Garnaes J, Tang P T 2015 Replication fidelity assessment of large area sub- $\mu \mathrm{m}$ structured polymer surfaces using scatterometry Surf. Topogr. Metrol. Prop. 3045005.

[14] Madsen MH, Hansen PE, Zalkovskij M, Karamehmedović M, Garnæs J 2015 Fast characterization of moving samples with nano-textured surfaces Optica 2301.

[15] Garnaes J, Kofod N, Kule A, Nielsen C, Dircherl K and Blunt L 2003 Calibration of step heights and roughness measurements with atomic force microscopes, Precision Engineering : 91-98.

[16] Garnaes J, Kühle A, Nielsen L, Borsetto F 2005 True three-dimensional calibration of closed loop scanning probe microscopes, Nanoscale Calibration Standards and Methods - Dimensional and Related 
Measurements in the Micro- and Nanometer Range Editors G. Wilkening and L. Koenders, Wiley-VCH Germany page 193-204.

[17] Image Metrology A/S Scanning Probe Image Processing (SPIPTM), http://www.imagemet.com/, 2015. [18] ISO 5436 part 1:200 2000 Geometrical product specification (gps) -surface texture: profile method measurement standard - material measures. Technical report, International Organization of Standardization.

[19] Joint Committee for Guides in Metrology (JCGM) 2008 JCGM 100:2008 Guide to the Expression of Uncertainty in Measurement (GUM). . i-viii, 1-132.

[20] Physikalische-Technische Bundesanstalt. Step height standard, shs type b 1000 c03 r17n337, certificate number PTB-5.2-4038842,2008.

[21] Tosello G, Hansen H N, Marinello F and Gasparin S 2010 Replication and dimensional quality control of industrial nanoscale surfaces using calibrated afm measurements and sem image processing, Annals of the CIRP, 59/1:563-568.

[22] Ito H and Suzuki H, 2009 Micro-Features Formation in Injection Compression Moulding, Journal of Solid mechanics and Material Engineering, 3/2,320-327.

[23] Suzuki H, Takayama T and Ito H 2012 Replication behaviour for micro surface features with high aspect ratio and structure development in injection compression moulding, Proceeding of Advanced Material Development and Performances, 6:563-569.

[24] Rohde M, Derdouri A and Kamal M.-R2009 Micro Replication by Injection-Compression Moulding, Conf. Proc. Polymer Processing 24th : 288-297.

[25] Leo V and Cuvelliez C 1996 The effect of the packing parameters, gate geometry, and mold elasticity on the final dimensions of a molded part Polymer Engineering and Science 36/15: 1961-1971.

[26] Matschuk M, Bruus H and Larsen N B 2010 Nanostructures for all-polymer microfluidic systems. Microelec. Eng. 87: 1379-1382.

[27] Griffiths C A, Dimov S S, Scholz S and Tosello G, 2011 Cavity air flow behaviour during filling in microinjection molding, Journal of Manufacturing Science and Engineering, 133, pp: 10.
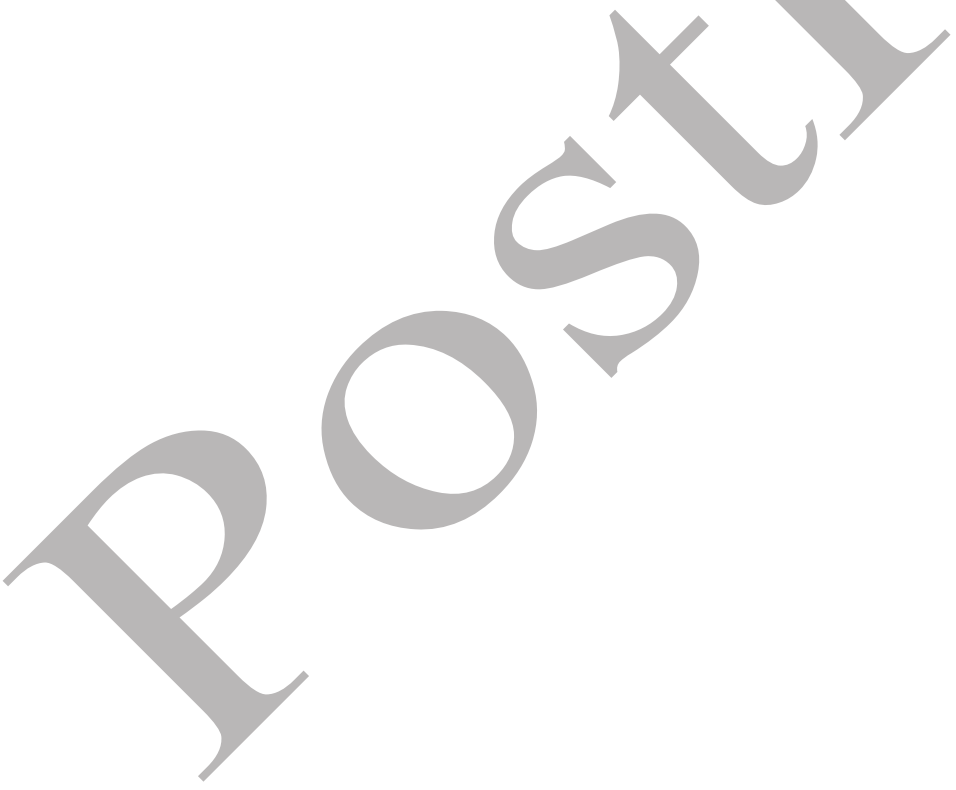